\title{
Usefulness of Monitoring Stroke Volume Variations for Fluid Management during Pediatric Living-Donor Liver Transplantation
}

\author{
Yoshihiro Kasagi $^{1}$, Manabu Hashimoto ${ }^{2}$, Shugo Kasuya ${ }^{2}$, Seisuke Sakamoto ${ }^{3}$, Mureo Kasahara ${ }^{3}$, \\ Yasuyuki Suzuki ${ }^{2}$, Eiichi Inada ${ }^{1}$ \\ ${ }^{1}$ Department of Anesthesiology and Pain Medicine, Juntendo University School of Medicine, Tokyo, Japan; ${ }^{2}$ Department of Anes- \\ thesia and Intensive Care, National Center for Child Health and Development, Tokyo, Japan; ${ }^{3}$ Transplantation Surgery, National \\ Center for Child Health and Development, Tokyo, Japan. \\ Email: yoshihirokasagi@hotmail.com
}

Received June $19^{\text {th }}, 2012$; revised July $22^{\text {nd }}$; accepted August $13^{\text {th }}, 2012$

\begin{abstract}
Purpose: Central venous pressure (CVP) is considered to be unsuitable as preload parameter. Stroke volume variation (SVV) has recently been reported to be effective as a preload and fluid responsiveness parameter, and its usefulness for fluid management during living-donor liver transplantation (LDLT). However, use of SVV has not been reported in children. Our aim is to evaluate the use of SVV as a target parameter of circulating blood volume during pediatric LDLT. Methods: This retrospective study was conducted in 40 consecutive patients aged between 5 and 109 months who underwent elective LDLT. Twenty patients underwent LDLT without FloTrac ${ }^{\mathrm{TM}}$ (C group) and the rest patients underwent LDLT with the FloTrac ${ }^{\mathrm{TM}}$ monitoring (F group). As a fluid management target, CVP was maintained at 10 $\mathrm{mmHg}$ in the C group and SVV at 10\% in the F group. We compared MAP and CVP at the times of the greatest decrease within 5 minutes after reperfusion. Results: MAP after reperfusion was significantly decreased in both groups $(\mathrm{P}<0.01)$, with the magnitude of decrease significantly greater in the $\mathrm{C}$ group compared with the $\mathrm{F}$ group $(\mathrm{P}=0.02)$. MAP before reperfusion did not significantly differ between the groups. After reperfusion, CVP was nearly the same in both groups, with that in the $\mathrm{C}$ group slightly decreased and nearly no change in the F group. SVV after reperfusion was significantly increased $(\mathrm{P}<0.001)$. Conclusion: When used as a target parameter for fluid management during pediatric LDLT, hemodynamic changes were less when SVV was used as the parameter of circulating blood volume.
\end{abstract}

Keywords: Pediatric; Living-Donor Liver Transplantation; Fluid Management; Stroke Volume Variation; Reperfusion

\section{Introduction}

Although circulating blood volume during living-donor liver transplantation (LDLT) must be carefully controlled, reliable methods have not been established [1]. Hemodynamic management and maintaining proper circulatory volume is a challenge in patients undergoing liver transplantation. Although pulmonary artery catheter and transesophageal echocardiography (TEE) may be used in adult patients, it may not be possible to use the pulmonary artery catheter or TEE. We must rely on the central venous pressure in infants and small children undergoing liver transplantation. Maintaining low central venous pressure (CVP) may be useful from the perspective of reducing infusion or transfusion volume, which can affect prognosis $[2,3]$. However, renal function may decline, thus increasing the risk of postoperative mortality
[4]. In addition, liver transplant recipients with hepatic failure have a bleeding tendency and often develop massive bleeding due to clotting abnormalities, thrombocytopenia due to hypersplenism, and collateral circulation. In particular, large hemodynamic changes are seen during reperfusion of the transplanted liver and insufficient circulating blood volume can result in marked hypotension, leading to dysfunction of the transplanted liver and poor prognosis [5]. Because excess fluids leads to complications, including pulmonary edema and organ congestion [1], evaluation of circulating blood volume is important.

As preload parameters, CVP and pulmonary artery occlusion pressure (PAOP) have been traditionally used, though no correlation has been noted between them due to changes in left ventricular end diastolic volume (LVEDV), cardiac output index, stroke index, and volume load. 
Thus, these parameters are considered to be unsuitable as preload parameters [6]. Stroke volume variation (SVV), as determined by continuous monitoring of the arterial pressure waveform, has recently been reported to be effective as a preload and fluid responsiveness parameter [7-10], and its usefulness for fluid management during liver transplantation has also been demonstrated $[9,10]$. However, to date, clinical use of SVV has been limited to adults, with very few reports of use in children, and none in regards to fluid management during liver transplantation.

In this study, we evaluated the use of SVV as a target parameter of circulating blood volume during pediatric LDLT. We compared our findings to those obtained with CVP in regard to usefulness as a parameter for hemodynamic status.

\section{Patients and Methods}

Informed consent was obtained by document from all patient families. This retrospective study was conducted with 40 consecutive patients aged between 5 and 109 months who underwent elective LDLT at National Center for Child Health and Development, Tokyo, Japan between September 2008 and December 2010. In July 2009, the FloTrac ${ }^{\mathrm{TM}}$ system (Edwards Lifesciences LLC, Irvine, CA, USA) was introduced to our hospital for measurement of SVV. The 40 patients included 20 who were treated before introduction of the FloTrac ${ }^{\mathrm{TM}}$ system (C group) and 20 patients who were treated after (F group).

All patients were premedicated with oral diazepam (Takeda Pharmaceutical Company Limited, Tokyo, Japan) at $0.7 \mathrm{mg} / \mathrm{kg}$. An infusion of remifentanil (Jansen Pharmaceutical K.K. Tokyo, Japan) $0.5 \mu \mathrm{g} / \mathrm{kg} / \mathrm{min}$ was started, then tracheal intubation was performed after administrations of midazolam (Astellas Pharma Inc., Tokyo, Japan) at $0.1-0.2 \mathrm{mg} / \mathrm{kg}$, fentanyl (Daiichi Sankyo Company, Limited, Tokyo, Japan) at $2 \mu \mathrm{g} / \mathrm{kg}$, and rocuronium (MSD K.K. Tokyo, Japan) at $1 \mathrm{mg} / \mathrm{kg}$. Anesthesia was maintained with propofol (Maruishi Pharmaceutical Company Limited, Osaka, Japan) at 60 - 90 $\mathrm{mg} / \mathrm{kg} / \mathrm{h}$ or inhaled sevoflurane at a concentration of $0.8 \%-1.5 \%$. In all patients, remifentanil was continuously administered at $0.25-1.0 \mu \mathrm{g} / \mathrm{kg} / \mathrm{min}$, with fentanyl and rocuronium intermittently administered.

Following tracheal intubation, an arterial pressure line and central venous catheter were placed. Arterial pressure was measured using a conventional pressure transducer in the $\mathrm{C}$ group and with the FloTrac ${ }^{\mathrm{TM}}$ system in the F group. During surgery, in addition to basic monitoring of invasive arterial pressure and CVP, SVV was also monitored in the F group. Fluids included bicarbonated Ringer's solution, acetated Ringer's solution, and $5 \%$ albumin solution. Blood transfusion products were administered as needed at the discretion of the attending anesthesiologist.
As a fluid management target, before reperfusion of the liver, CVP was maintained at $\geq 10 \mathrm{mmHg}$ in the $\mathrm{C}$ group and SVV at $\leq 10 \%$ in the $\mathrm{F}$ group. After reperfusion was started, intravenous phenylephrine at $5 \mu \mathrm{g} / \mathrm{kg}$ was given when the mean blood pressure (MAP) decreased by more than $40 \%$ of the preperfusion levels. Intraoperative variables included the number of administrations of phenylephrine within 5 minutes of starting reperfusion, and MAP and CVP at the times of the greatest decrease before starting and within 5 minutes after starting reperfusion. These were compared between the two groups. A t-test, Mann-Whitney's U-test and $\chi^{2}$ test were used for statistical analyses.

\section{Results}

The mean operative time in the $\mathrm{C}$ group including 2 patients with operation times over 750 minutes was significantly longer as compared with the $\mathrm{F}$ group $(\mathrm{P}=$ 0.018). No other significant differences were identified between the groups, including age, anesthesia time, and fluid balance (Table 1). The mean number of administrations of phenylephrine in the $\mathrm{C}$ group $(0.9 \pm 0.8)$ was about twice that in the $\mathrm{F}$ group $(0.4 \pm 0.7, \mathrm{P}=0.19)$, without significance. MAP after reperfusion was significantly decreased in both groups $(\mathrm{P}<0.01)$, with the magnitude of decrease significantly greater in the $\mathrm{C}$ group $(-17 \pm 7 \mathrm{mmHg})$ compared with the $\mathrm{F}$ group $(-11 \pm 7$ $\mathrm{mmHg}, \mathrm{P}=0.02$ ). MAP before and after reperfusion did not significantly differ between the groups (Table 2). Before reperfusion, CVP was significantly higher in the $\mathrm{C}$ group than in the $\mathrm{F}$ group $(\mathrm{P}=0.03)$. After reperfusion, CVP was nearly the same in both groups, with that in the $\mathrm{C}$ group slightly decreased and nearly no change in the $\mathrm{F}$ group. SVV after reperfusion was significantly increased in the $\mathrm{F}$ group $(\mathrm{P}<0.001)$. There was 1 postoperative death in the $\mathrm{C}$ group and 2 in the $\mathrm{F}$ group, though the difference was not significant.

Table 1. Patients' characteristics.

\begin{tabular}{lcc}
\hline & C group $(\mathrm{n}=20)$ & $\mathrm{F}$ group $(\mathrm{n}=20)$ \\
\hline Age (months) & $25.5 \pm 30.8$ & $28.3 \pm 28.8$ \\
Sex (male/female) & $7 / 3$ & $6 / 14$ \\
Weight $(\mathrm{kg})$ & $12.1 \pm 8.0$ & $9.8 \pm 4.4$ \\
Diagnosis (biliary atresia: others) & $7 / 3$ & $6 / 14$ \\
Duration of anesthesia (min) & $674 \pm 120$ & $605 \pm 94$ \\
Duration of surgery (min) & $558 \pm 110^{*}$ & $479 \pm 81$ \\
Fluids $(\mathrm{ml})$ & $2326 \pm 967$ & $2022 \pm 716$ \\
Transfusion $(\mathrm{ml})$ & $436 \pm 588$ & $482 \pm 473$ \\
$5 \%$ Albumin $(\mathrm{ml})$ & $1402 \pm 818$ & $779 \pm 406$ \\
Blood loss $\left(\mathrm{g} \cdot \mathrm{kg}^{-1}\right)$ & $92 \pm 90$ & $104 \pm 106$ \\
Fluid balance $\left(\mathrm{ml} \cdot \mathrm{kg}^{-1} \cdot \mathrm{h}^{-1}\right)$ & $13.4 \pm 4.9$ & $15.7 \pm 5.3$ \\
\hline
\end{tabular}

Data are presented as the mean \pm standard deviation or number of patients. Water balance: (fluids + transfusion) - (blood loss + urine output) $;{ }^{*} \mathrm{P}<0.05$ in comparison with the $\mathrm{F}$ group $(\mathrm{P}=0.015)$. 
Table 2. Hemodynamic changes before and after reperfusion.

\begin{tabular}{lcccc}
\hline & \multicolumn{2}{c}{ C group $(\mathrm{n}=20)$} & \multicolumn{2}{c}{ F group $(\mathrm{n}=20)$} \\
\cline { 2 - 5 } & before & after & before & after \\
\hline MAP (mmHg) & $67 \pm 11$ & $50 \pm 9^{* *}$ & $67 \pm 12$ & $56 \pm 10^{* *}$ \\
CVP (mmHg) & $14 \pm 4$ & $11 \pm 4$ & $11 \pm 4^{\dagger}$ & $10 \pm 3$ \\
SVV (\%) & & & $6 \pm 1$ & $12 \pm 6^{* *}$ \\
\hline
\end{tabular}

Data are presented as the mean \pm standard deviation; ${ }^{* *} \mathrm{P}<0.01$ in comparison with respective value before reperfusion; ${ }^{\dagger} \mathrm{P}<0.05$ in comparison with the $\mathrm{C}$ group.

\section{Discussion}

We compared hemodynamic parameters before and after reperfusion in children who underwent LDLT during anesthesia using CVP or SVV as the target parameter for circulating blood volume. When SVV was used as a target, despite a lack of differences in the amount of the intraoperative fluid and transfused blood products, CVP was significantly lower before reperfusion. Conversely, after reperfusion, the number of administrations of phenylephrine was less and MAP tended to remain high. In addition, SVV was significantly increased and MAP significantly decreased after reperfusion, whereas CVP was not significantly changed. These results suggest that CVP does not accurately reflect circulating blood volume, because it is easily affected by surgical manipulations such as compression or blocking of the inferior vena cava. During the perioperative period of various types of surgery, including liver transplantation, SVV has been reported to represent a more reliable preload parameter in adults as compared to CVP $[7,8]$. Our study confirmed those findings during pediatric LDLT. Although CVP did not show large changes, SVV was significantly elevated after reperfusion. This finding may suggest that SVV is more useful than CVP in this procedure.

SVV has previously been used as a marker of fluid responsiveness for cases in which a decrease in circulating blood volume is anticipated [7-10], and hemodynamic improvement with fluid loading has been reported when SVV is $\geq 8 \%-10 \%[9,10]$. In the present study, as previously reported by Biais et al. [9], the target value for SVV before reperfusion was $\leq 10 \%$ and was maintained at $\leq 8 \%$ in all patients. We considered that maintaining SVV at a relatively low level as compared to CVP as a target parameter was likely one of the reasons why hemodynamics after reperfusion were more stable. Nevertheless, suitable SVV values during liver transplantation require further investigation.

Attempts at measuring cardiac output based on arterial pressure waveform analysis have been reported [11].
However, a key disadvantage is the large intra-individual and inter-individual differences in those relationships, and external calibration with single lithium dilution $\left(\mathrm{LiDCO}^{\mathrm{TM}}\right)$ or thermodilution $\left(\mathrm{PiCCO}^{\mathrm{TM}}\right)$ is required $[12,13]$. A major feature of the FloTrac ${ }^{\mathrm{TM}}$ system, used to measure SVV in our study, is that cardiac output, stroke volume, and SVV are easily measured by connecting a special sensor (FloTrac ${ }^{\mathrm{TM}}$; Edwards Lifesciences LLC) and monitor (Vigileo ${ }^{\mathrm{TM}}$; Edwards Lifesciences LLC) to the arterial line, without the need for a central venous catheter or pulmonary artery catheter, or external calibration [14].

With the FloTrac ${ }^{\mathrm{TM}}$ system, in addition to SVV, cardiac output and stroke volume are also calculated. However, since the constant use in the calculation process varies with age and arterial compliance is high in young individuals, even when a large change in cardiac output occurs, little change is seen in the arterial pressure waveform [15]. Thus, these parameters are less reliable in patients $\leq 20$ years old.

SVV is expressed by the following formula using maximum stroke volume $\left(\mathrm{SV}_{\max }\right)$ and minimum stroke volume $\left(\mathrm{SV}_{\min }\right)$, standard deviations $\left(\mathrm{SD}_{\max }\right.$ and $\left.\mathrm{SD}_{\min }\right)$, and the constant $\chi$ determined by vascular compliance and vascular resistance [16].

$$
\begin{aligned}
\mathrm{SVV} & =\left(\mathrm{SV}_{\max }-\mathrm{SV}_{\text {min }}\right) /\left(\mathrm{SV}_{\text {max }}+\mathrm{SV}_{\text {min }}\right) / 2 \\
& =\left(\chi \cdot \mathrm{SD}_{\text {max }}-\chi \cdot \mathrm{SD}_{\text {min }}\right) /\left(\chi \cdot \mathrm{SD}_{\text {max }}+\chi \cdot \mathrm{SD}_{\text {min }}\right) / 2 \\
& =\left(\mathrm{SD}_{\text {max }}-\mathrm{SD}_{\text {min }}\right) /\left(\mathrm{SD}_{\text {max }}+\mathrm{SD}_{\text {min }}\right) / 2
\end{aligned}
$$

As shown in the above formula, SVV is calculated only from changes in stroke volume and, theoretically, should be applicable for all ages. Although not previously reported in pediatric cases, our findings suggest that SVV may be a useful target parameter for intraoperative fluid management during LDLT in children. A future study is needed to validate the usefulness of monitoring SVV in children. Whether SVV is useful for pediatric before SVV can be proposed as a useful target parameter for fluid management in pediatric surgery cases.

Also, because of its retrospective nature and the different time periods when surgery was performed between the groups, some influence due to differences in surgical techniques cannot be excluded. In addition, blood transfusions and albumin administration were performed at the discretion of the attending anesthesiologist, which may have also contributed to the lack of significant differences. In the future, a prospective study with a more detailed protocol should be conducted.

In conclusion, hemodynamic changes associated with reperfusion were less when SVV was used as a target parameter of circulating blood volume. SVV was associated with hemodynamic changes before and after starting 
reperfusion as compared to CVP.

\section{REFERENCES}

[1] Y. Ozier and J. R. Klinck, "Anesthetic Management of Hepatic Transplantation," Current Opinion in Anesthesiology, Vol. 21, No. 3, 2008, pp. 391-400. doi:10.1097/ACO.0b013e3282ff85f4

[2] M. T. de Boer, M. C. Christensen, M. Asmussen, C. S. van der Hilst, H. G. Hendriks, M. J. Slooff, et al., "The Impact of Intraoperative Transfusion of Platelets and Red Blood Cells on Survival after Liver Transplantation," Anesthesia \& Analgesia, Vol. 106, No. 1, 2008, pp. 32-44. doi:10.1213/01.ane.0000289638.26666.ed

[3] L. Massicotte, S. Lenis, L. Thibeault, M. P. Sassine, R. F. Seal and A. Roy, "Effect of Low Central Venous Pressure and Phlebotomy on Blood Product Transfusion Requirements during Liver Transplantations," Liver Transplantation, Vol. 12, No. 1, 2006, pp. 117-123. doi:10.1002/lt.20559

[4] R. A. Schroeder, B. H. Collins, E. Tuttle-Newhall, K. Robertson, J. Plotkin, L. B. Johnson, et al., "Intraoperative Fluid Management during Orthotopic Liver Transplantation," Journal of Cardiothoracic and Vascular Anesthesia, Vol. 18, No. 4, 2004, pp. 438-441. doi:10.1053/j.jvca.2004.05.020

[5] D. L. Reich, R. K. Wood Jr., S. Emre, C. A. Bodian, S. Hossain, M. Krol, et al., "Association of Intraoperative Hypotension and Pulmonary Hypertension with Adverse Outcomes after Orthotopic Liver Transplantation," Journal of Cardiothoracic and Vascular Anesthesia, Vol. 17, No. 6, 2003, pp. 699-702. doi:10.1053/j.jvca.2003.09.010

[6] A. Kumar, R. Anel, E. Bunnell, K. Habet, S. Zanotti, S. Marshall, et al., "Pulmonary Artery Occlusion Pressure and Central Venous Pressure Fail to Predict Ventricular Filling Volume, Cardiac Performance, or the Response to Volume Infusion in Normal Subjects," Critical Care Medicine, Vol. 32, No. 3, 2004, pp. 691-699. doi:10.1097/01.CCM.0000114996.68110.C9

[7] D. A. Reuter, T. W. Felbinger, C. Schmidt, E. Kilger, O. Goedje, P. Lamm, et al., "Stroke Volume Variations for Assessment of Cardiac Responsiveness to Volume Loading in Mechanically Ventilated Patients after Cardiac Surgery," Intensive Care Medicine, Vol. 28, No. 4, 2002, pp. 392-398. doi:10.1007/s00134-002-1211-z

[8] M. Kobayashi, M. Koh, T. Irinoda, E. Meguro, Y. Ha- yakawa and A. Takagane, "Stroke Volume Variation as a Predictor of Intravascular Volume Depression and Possible Hypotension during the Early Postoperative Period after Esophagectomy," Annals of Surgical Oncology, Vol. 16, No. 5, 2009, pp. 1371-1377. doi:10.1245/s10434-008-0139-0

[9] M. Biais, K. Nouette-Gaulain, V. Cottenceau, P. Revel and F. Sztark, "Uncalibrated Pulse Contour-Derived Stroke Volume Variation Predicts Fluid Responsiveness in Mechanically Ventilated Patients Undergoing Liver Transplantation," British Journal of Anaesthesia, Vol. 101, No. 6, 2008, pp. 761-768. doi:10.1093/bja/aen277

[10] Y. H. Shin, J. S. Ko, M. S. Gwak, G. S. Kim, J. H. Lee and S. K. Lee, "Utility of Uncalibrated Femoral Stroke Volume Variation as a Predictor of Fluid Responsiveness during the Anhepatic Phase of Liver Transplantation," Liver Transplantation, Vol. 17, No. 1, 2011, pp. 53-59. doi:10.1002/lt.22186

[11] R. R. Williams, R. B. Wray, T. J. Tsagaris and H. Kuida, "Computer Estimation of Stroke Volume from Aortic Pulse Contour in Dogs and Humans," Cardiology, Vol. 59, No. 6, 1974, pp. 350-366. doi:10.1159/000169694

[12] T. T. Hamilton, L. M. Huber and M. E. Jessen, "PulseCO: A Less-Invasive Method to Monitor Cardiac Output from Arterial Pressure after Cardiac Surgery," The Annals of Thoracic Surgery, Vol. 74, No. 4, 2002, pp. S1408-S1412. doi:10.1016/S0003-4975(02)04059-6

[13] G. D. Rocca, M. G. Costa, L. Pompei, C. Coccia and P. Pietropaoli, "Continuous and Intermittent Cardiac Output Measurement: Pulmonary Artery Catheter versus Aortic Transpulmonary Technique," British Journal of Anaesthesia, Vol. 88, No. 3, 2002, pp. 350-356. doi:10.1093/bja/88.3.350

[14] G. R. Manecke, "Edwards FloTrac Sensor and Vigileo Monitor: Easy, Accurate, Reliable Cardiac Output Assessment Using the Arterial Pulse Wave," Expert Review of Medical Devices, Vol. 2, No. 5, 2005, pp. 523-527. doi: $10.1586 / 17434440.2 .5 .523$

[15] F. Michard, "Changes in Arterial Pressure during Mechanical Ventilation," Anesthesiology, Vol. 103, 2005, pp. 419-428. doi:10.1097/00000542-200508000-00026

[16] M. Frederic, B. Sandrine, C Denis, A. Nadia, M. Alain, T. Jean-Louis, et al., "Relation between Respiratory Changes in Arterial Pulse Pressure and Fluid Responsiveness in Septic Patients with Acute Circulatory Failure," Critical Care Medicine, Vol. 162, No. 1, 2000, pp. 134-138. 